\title{
Epicutaneous immunotherapy protects cashew sensitized mice from anaphylaxis
}

\author{
Benjamin Pelletier ${ }^{1}$, Audrey Perrin ${ }^{1}$, Noémie Assoun ${ }^{1}$, Camille Plaquet ${ }^{1}$, Nathalie Oreal ${ }^{1}$, \\ Laetitia Gaulme $^{1}$, Adeline Bouzereau ${ }^{1}$, Jean-Louis Labernardière ${ }^{1}$, Mélanie Ligouis ${ }^{1}$, \\ Sophie Wavrin ${ }^{1}$, Katie Matthews ${ }^{1}$, Fabrice Porcheray ${ }^{1}$, Hugh Sampson ${ }^{1}$, and Pierre-Louis \\ Hervé $^{1}$
}

${ }^{1} \mathrm{DBV}$ Technologies

July 13, 2020

\begin{abstract}
Background: The prevalence of tree nut allergy has increased worldwide, and cashew has become one of the most common food allergens. More critically, cashew allergy is frequently associated with severe anaphylaxis. Despite the high medical need, no approved treatment is available and strict avoidance and preparedness for prompt treatment of allergic reactions are considered dual standard of care. In the meantime, Phase III study results suggest investigational epicutaneous immunotherapy (EPIT) may be a relevant and safe treatment for peanut allergy and may improve the quality of life for many peanut allergic children. Objective: We aimed to evaluate the capacity of EPIT to provide protection against cashew-induced anaphylaxis in a relevant mouse model. Methods: A mouse model of IgE-mediated cashew anaphylaxis was first developed. Based upon this model, the efficacy of EPIT was evaluated by applying patches containing cashew allergens to cashew-sensitized mice. Cashew-specific antibody titers were measured throughout treatment. Treated mice were challenged orally to cashew and anaphylactic symptoms were monitored. Additionally, plasma levels of mast-cell proteases (mMCP)-1/7 were quantified from blood samples collected after challenge to evaluate IgE-induced mast-cell activation. Results: EPIT significantly decreased anaphylactic symptoms following challenge and increased cashew-specific IgG2a (equivalent of human IgG1). Interestingly, this protection was associated with a sharp decrease in mast-cell reactivity. Conclusion: We demonstrate that EPIT markedly reduced IgE-mediated allergic reactions in a mouse model of cashew allergy, which suggests that EPIT may be a relevant approach to treating cashew allergy.
\end{abstract}

\section{Introduction}

The cashew plant (Anacardium occidentale) is a tropical evergreen tree belonging to the Anacardiaceae family, which includes mango and pistachio. It produces cashew seeds (nuts) that are regularly consumed by most of the world's population. Unfortunately, cashew is also classified as one of the most potent allergenic food, and no approved specific treatment is available to address this issue. ${ }^{1}$ The prevalence of cashew allergy has risen over the last decades in industrial countries with the increasing consumption of this nut. ${ }^{2,3}$ Besides the prevalence, a high medical need for a treatment is warranted by the unique severity of anaphylactic reactions triggered by the consumption of cashew-containing food in allergic individuals. ${ }^{4,5}$ Consequently, safe treatments minimizing contact between cashew allergens and anaphylaxis-triggering effector cells, such as mast-cells, should be given paramount considerations. Investigational epicutaneous immunotherapy (EPIT) is under review by the U.S. FDA for peanut allergy treatment in children. ${ }^{6}$ This epicutaneous system is comprised of an allergen-adsorbed patch, held above the intact skin by a circular crown of adhesive foam to create an occlusive condensation chamber (Viaskin). This technology permits allergen solubilization by transepidermal water loss and promotes its delivery across the stratum corneum to epidermal Langerhans cells. $^{7}$ This route of administration is a key element of the safety profile of EPIT, making it a relevant 
approach for the treatment of life-threatening allergies such as cashew. ${ }^{8}$ In that context, the aim of the present work was to assess the capacity of EPIT to protect against anaphylaxis in a mouse model of cashew allergy. To that end, a mouse model of IgE-mediated cashew anaphylaxis was first developed. Then, the ability of patches to deliver cashew allergens to skin dendritic cells was demonstrated. Finally, cashewsensitized mice were treated with cashew patches (EPIT) for up to 16 weeks to measure the kinetic induction of specific antibodies and the level of protection afforded against anaphylaxis following oral challenge. Results showed that EPIT was able to significantly increase the level of cashew specific IgG2a (mouse equivalent of human IgG1) all along the therapy period. More importantly, EPIT mice were significantly protected against anaphylactic symptoms following oral challenge. Interestingly, this protection was associated with a strong decrease in the activation of mast-cells, which are the main immune effectors involved in IgE-mediated anaphylaxis.

\section{Methods}

\section{Animals and ethic.}

BALB/c mice were purchased from Charles River (Lyon, France) and housed under conventional conditions (DBV Technologies, Montrouge, France, agreement number \#A92-049-02). Experiments have been performed according to the European Community rules of animal care, and with permission of the French government (authorization \#13305).

\section{Extraction of cashew allergens for sensitization and challenge.}

Proteins were extracted from acetone-defatted cashew flour (Stallergenes Greer, Lenoir, NC) by overnight stirring in PBS $1 \mathrm{X}$, at $4^{\circ} \mathrm{C}$. The solution was centrifuged for $30 \mathrm{~min}$ at $3000 \mathrm{x}$ g to eliminate insoluble components. Supernatant was frozen at $-80^{\circ} \mathrm{C}$ and lyophilized (Lyofal, Salon de Provence, France). Nitrogen inertization was performed. Solutions for sensitization and oral challenge were obtained by adding the required volume of PBS $1 \mathrm{X}$ into vials. The final protein concentration was controlled using Bradford.

\section{Sensitization of mice.}

For oral sensitization, mice received $1 \mathrm{mg}$ of cashew protein extract supplemented with $10 \mu \mathrm{g}$ of Cholera Toxin (List Biological Laboratories) per intragastric route, at a rate of one intragastric administration per week for 6 consecutive weeks. For skin sensitization, mice received $100 \mu \mathrm{g}$ of cashew protein extract on the skin of the lower back. A liquid solution was deposited on a gauze that was maintained on depilated skin with Tegaderm ${ }^{\circledR}(3 \mathrm{M})$ for 72 hours. Before each application, skin was pre-treated using a dermatological laser (Pantec Biosolutions) at a fluence of $5.5 \mathrm{Joules} / \mathrm{cm}^{2}$ to mimic atopic skin. Mice received allergen twice a week, every two weeks, for 7 weeks.

\section{Injection of anti IgE and IgG receptor blocking antibodies.}

Mice received $100 \mu \mathrm{g}$ of anti-IgE (clone EM-95, kindly provided by Fred Finkelman), $100 \mu \mathrm{g}$ of antiFcrRII/RIII (clone 2.4G2, Bio X Cell) or a mix of rat IgG2b and rat IgG2a as isotype controls (clones LTF-2 and 2A3, respectively, Bio X Cell) by intraperitoneal route. To avoid any non-specific anaphylactic reaction, all mice received $200 \mu \mathrm{g}$ of triprolidine hydrochloride (Sigma) by intraperitoneal route 30 minutes before injection of the monoclonal antibodies. Mice were challenged orally 24 hours later.

\section{Preparation of cashew patches and application to mice.}

Epicutaneous patches were loaded with $50 \mu \mathrm{g}$ of cashew protein extract (Stallergenes Greer, Lenoir, NC) or $50 \mu \mathrm{g}$ of cashew protein extract conjugated to Fluoroprobe-647 (Interchim), prepared in phosphate buffer $0.1 \mathrm{M}$. Patches were dried at $30^{\circ} \mathrm{C}$ in a ventilated oven and stored at $4^{\circ} \mathrm{C}$. Before patch application, mice were anaesthetized with ketamine and xylazine (50 and $10 \mathrm{mg} / \mathrm{kg}$, respectively) and hair on the back was removed using electric clippers and depilatory cream (Reckitt Benckiser). Patches were applied the following day and secured using an Urgoderm ${ }^{\circledR}$ bandage (Urgo Laboratories) for 48 hours. As a negative control, mice received patches loaded with phosphate buffer $0.1 \mathrm{M}$. 


\section{Collection of brachial lymph nodes (BLNs) for flow cytometry analysis.}

BLNs were harvested in $2 \mathrm{~mL}$ of RPMI containing $0.26 \mathrm{U} / \mathrm{mL}$ Liberase TL and $25 \mu \mathrm{g} / \mathrm{mL}$ DNase I (Sigma Aldrich). Each BLN was flushed using a syringe and incubated $20 \mathrm{~min}$ at $37^{\circ} \mathrm{C}$. The enzymatic reaction was then stopped with $250 \mu \mathrm{L}$ of EDTA $100 \mathrm{mM}$. Cells were homogenized with a $100 \mu \mathrm{m}$ cell strainer in magneticactivated cell sorting buffer (Miltenyi Biotec) and counted. Cells were incubated for 15 min at $4^{\circ} \mathrm{C}$ with $\mathrm{Fc}$ Block (BD Biosciences) and stained for 25 minutes at $4^{\circ} \mathrm{C}$ with fluorochrome-conjugated antibodies: antiCD11c-PE (clone REA754, Miltenyi Biotec), anti-MHC-II-VioBlue (clone REA813, Miltenyi Biotec), antiCD11b-PerCP-Vio700 (clone REA592, Miltenyi Biotec), anti-EpCAM-PE-Vio770 (clone caa7-9G8, Miltenyi Biotec), anti-XCR1-APC-Vio700 (clone REA707, Miltenyi Biotec). Additionally, dead cells were excluded with Zombie Aqua (Biolegend) staining. Cells were acquired on MACSquant 10 flow cytometer (Miltenyi Biotec) and data were analyzed using FlowJo software using the gating strategy previously described. ${ }^{9}$

\section{Collection of plasma.}

Blood samples were collected by submandibular puncture into microtubes containing EDTA (MiniCollect, Greiner Bio-One) and centrifugated at $3000 \mathrm{x}$ g for 10 minutes to collect plasma. Plasma samples were stored at $-20^{\circ} \mathrm{C}$.

\section{Measurement of antibody titers by ELISA.}

IgG1 and IgG2a titers were measured by direct ELISA: 96-well plates were coated overnight at $4^{\circ} \mathrm{C}$ with cashew protein extract $(5 \mu \mathrm{g} / \mathrm{mL}$, protein equivalent) or with anti-IgG1 or anti-IgG2a $(5$ or $1 \mu \mathrm{g} / \mathrm{mL}$, respectively) for binding of standards. Plates were washed in PBS $1 \mathrm{X}$-Tween ${ }^{\circledR} 0.05 \%$ and blocked 1 hour at room temperature (RT) with phosphate $0.1 \mathrm{M} \mathrm{pH} 7.4-\mathrm{NaCl} 0.15 \mathrm{M}$-BSA $0.1 \%$-sodium azide $0.01 \%$ (EIA buffer). Plates were then incubated overnight at $4^{\circ} \mathrm{C}$ with mouse plasma (in duplicates) or serially diluted standards (Rabbit F(ab')2 anti-mouse IgG1 or Human anti-mouse IgG2a, Bio-Rad), and then 1 hour at $37^{\circ} \mathrm{C}$ with relevant secondary antibodies conjugated to alkaline phosphatase (AP) (Bio-Rad). Finally, plates were incubated for 30 minutes at RT with AP substrate (p-Nitrophenyl Phosphate, pNPP) and optical densities (O.D.) at $405 \mathrm{~nm}$ were recorded. Antibody concentrations were calculated with Boltzmann sigmoidal equation using O.D. obtained from standards (GraphPad Prism ${ }^{\circledR}$ ), and after subtracting O.D. obtained from non-specific points. IgE titers were measured by indirect ELISA to avoid competition with IgG1 binding: 96-well plates were coated with $2 \mu \mathrm{g} / \mathrm{mL}$ of rat anti-mouse IgE (Bio-Rad) and incubated overnight at $4^{\circ} \mathrm{C}$. Plates were washed in PBS 1X-Tween ${ }^{\circledR} 0.05 \%$ and blocked 1 hour at RT with EIA buffer. Following washing, plates were incubated overnight at $4^{\circ} \mathrm{C}$ with mouse plasma (in duplicates) or serially diluted standard (mouse IgE, Bio-Rad), and then 1 hour at RT with biotinylated cashew proteins for plasma samples or biotin antimouse IgE (1:2000, Biolegend) for standard. Plates were finally incubated 1 hour at RT with AP-conjugated streptavidine (1:5000, Jackson Immuno Research Lab), and then 15 minutes at RT with pNPP. O.D. were recorded and antibody concentrations were measured as described above.

\section{Oral challenge and monitoring of anaphylaxis.}

Mice were challenged orally with $45 \mathrm{mg}$ of cashew protein extract (protein equivalent). Anaphylactic reactions were characterized by a drop in body temperature and the occurrence of clinical symptoms. Body temperature was measured before challenge and every 5 minutes for 60 minutes following challenge using subcutaneous transponders (Plexx). Clinical symptoms were recorded following the same frequency.Figure 2 : diarrhea was scored as followed: 0 , no diarrhea; 1 , soft but well-shaped stool; 2 , soft and unformed stool; 3 , one episode of diarrhea; 4, at least two episodes of diarrhea. Figure $\mathbf{5}$ : general symptoms were scored as followed: 0 , no symptoms; 1, repetitive nose scratching and ruffled fur; 2, mouse and eyes swelling, pilar erectus and reduced activity; 3 , difficulty breathing, mouth cyanosis and diarrhea; 4, no reaction following stimulation of whiskers, tremor and/or seizure; 5 , death. Mice that reached a general symptom score of 4 were sacrificed in agreement with ethical guidelines.

\section{Measurement of mMCP-1 and mMCP-7 by ELISA.}

96-well plates were coated overnight at $4^{\circ} \mathrm{C}$ with anti-mouse MCPT-1 (eBioscience) or MCPT-7 (R\&D 
systems). Plates were washed in PBS $1 \mathrm{X}$-Tween ${ }^{\circledR} 0.05 \%$ and blocked 1 hour at RT with blocking buffer 1X (eBioscience). Plates were incubated overnight at $4^{\circ} \mathrm{C}$ with plasma samples or recombinant MCPT-1 (eBioscience) or MCPT-7 (R\&D systems) as standards. Following washing, plates were incubated 1 hour at RT with biotinylated anti-mouse MCPT-1 (eBioscience) or MCPT-7 (R\&D systems), then 30 minutes at RT with HRP-conjugated avidin (eBioscience). HRP was developed using TMB substrate (eBioscience), for 15 minutes at RT. The reaction was stopped with $1 \mathrm{~N}$ chloride acid and O.D. at $450 \mathrm{~nm}$ was recorded. Concentrations were calculated with Boltzmann sigmoidal equation using O.D. obtained from standards (GraphPad Prism $\left.{ }^{\circledR}\right)$, and after subtracting O.D. obtained from non-specific points.

\section{Statistical analysis.}

Data are presented as median with interquartile ranges or mean with SEM. The non-parametric MannWhitney test was used to compare unpaired values (GraphPad Prism ${ }^{\circledR}$ ). Values of $\mathrm{p}<0.05$ were considered significant. The level of significance is indicated with asterisks: ${ }^{*}, \mathrm{p}<0.05 ;{ }^{* *}, \mathrm{p}<0.01 ;{ }^{* * *}, \mathrm{p}<0.001 ;{ }^{* * * *}$, $\mathrm{p}<0.0001$ and n.s., non-significant.

\section{Results}

\section{Development of a mouse model of cashew anaphylaxis.}

First, our cashew protein extraction method was characterized by analyzing subsequent protein extracts on SDS-PAGE (Figure S1 ). The obtained electrophoretic profile was identical to that of the initial cashew flour as well to that of commercial cashew protein extracts. Moreover, major allergens (Ana o 1/2/3) were retrieved at molecular weights and proportions identical to what has been described previously. ${ }^{10,11}$ Using this extract, two routes of sensitization were investigated: oral and skin. The evaluation of skin as a sensitization route was prompted by previous data suggesting that skin is a proposed portal of entry for peanut allergens, especially in patients suffering from atopic dermatitis. ${ }^{12}$ Here, mice were treated by laser microporation to mimic eczematous skin before topical application of allergens. Both routes of sensitization induced cashew-specific IgE, IgG1 and IgG2a (Figure 1A ). However, IgE titers were significantly higher in mice sensitized orally. Conversely, IgG2a titers were significantly higher in mice sensitized cutaneously. Following oral challenge, anaphylactic reactions were similar between the two routes (Figures $\mathbf{1 B}$ and $\mathbf{1 C}$ ). Importantly, both routes were able to promote mast-cell degranulation following oral challenge, as evidenced by a significant increase in mMCP-1 and mMCP-7 levels. However, the greatest increases were observed in orally sensitized mice (Figures 1D and 1E ). There is strong evidence for IgE-dependent anaphylaxis and little evidence for IgG-dependent anaphylaxis in humans. Conversely, a consistent role for IgG in anaphylaxis has been demonstrated in mice. ${ }^{13}$ Thus, we considered the oral route as the most clinically relevant since it gave the surest indicators of IgE-mediated anaphylaxis (highest IgE titers and strongest mast-cell activation). To validate this, orally sensitized mice received anti-IgE or anti-IgG blocking antibodies 24 hours prior to oral challenge. As expected, anti-IgE was able to limit anaphylactic symptoms and significantly reduced mast-cell degranulation (Figure 2 ). Although anti-IgG was also able to reduce temperature drop (Figure $\mathbf{2 A}$ ), it had no impact on diarrhea nor mast-cell activation (Figures $\mathbf{2 B}, \mathbf{2 C}$ and $\mathbf{2 D}$ ). Overall, these data confirmed that $\operatorname{IgE}$ is the main mediator of anaphylaxis in orally sensitized mice, but that IgG is also involved, albeit to a more modest level.

\section{Development and validation of cashew patches.}

To identify the optimal excipient for patch manufacturing, lyophilized cashew protein extract was solubilized in either PBS 1X, $\mathrm{NaCl} 0.45 \%$ or phosphate buffer $0.1 \mathrm{M}$ and deposited on epicutaneous patches. Deposits were dried and then resolubilized using distilled water and analyzed by ELISA or SDS PAGE (Figure S2 ). Cashew-specific IgG1 or IgE generated in orally sensitized mice reacted with all extract deposits, demonstrating that their immunogenicity was preserved (Figure S2A ). Moreover, the electrophoretic profiles of all deposits were comparable to that of the initial protein extract (Figure S2B ). However, the best protein integrity was obtained with phosphate buffer, as demonstrated by the absence of aggregated proteins in the pellet, that was selected as the preferred excipient. Patches loaded with fluorescent cashew proteins were applied to cashew-sensitized mice for 48 hours to evaluate the capacity of cashew patches to deliver allergens 
to skin dendritic cells (DCs). To control for the degree of sensitization, cashew-specific IgG1, IgG2a and IgE were quantified from plasma collected before patches were applied. All mice presented similar antibody titers that were consistent with previous experiments (data not shown). The number of fluorescent DCs was then measured from BLNs (Figure 3 ). A significant increase of cashew-positive DCs was observed in mice that received cashew patches compared to those receiving excipient patches. Of note, this increase was more pronounced in cDC2 dermal DCs and Langerhans cells than in cDC1 dermal DCs. Overall, these data demonstrate that cashew patches can be produced while maintaining allergen integrity and that they are able to deliver allergens to skin DCs, especially Langerhans cells and cDC2.

\section{EPIT with cashew patches increased cashew-specific IgG2a in sensitized mice .}

Mice were sensitized orally to cashew as described above and received cashew patches for up to 16 weeks, at a rate of 1 patch per week for 48 hours per application (EPIT). Plasma was collected every two weeks during treatment to measure the evolution of cashew-specific IgE, IgG1 and IgG2a (Figure 4 ). A transient increase of cashew-specific IgE was observed in EPIT mice compared to the sham group, with a peak level at week 12 post-treatment followed by a significant decrease (Figure 4A ). Similarly, an increase of cashew-specific IgG1 was observed in EPIT mice, with a peak level at week 10 post-treatment followed by a significant decrease (Figure 4B ). Finally, a progressive and continuous increase in cashew specific IgG2a was observed in treated mice compared to the sham group (Figure 4C). Overall, these data indicate that EPIT to cashew can strongly modulate cashew-specific antibody responses.

\section{EPIT with cashew patches protects sensitized mice against IgE-mediated anaphylaxis .}

Mice were sensitized and treated as described above. At the end of the treatment period (i.e. 8, 12 or 16 weeks), mice were challenged orally to cashew (Figure 5 ). A clear protective effect was observed in EPIT mice compared to the sham group and this protective effect was further improved with increasing length of treatment. This protection was characterized by a decrease in both temperature drop (Figures 5A, 5C and $\mathbf{5 E}$ ) and clinical symptoms (Figures $\mathbf{5 B}, \mathbf{5 D}$ and $\mathbf{5 F}$ ). To evaluate the capacity of EPIT to protect against mast-cell activation, plasma was collected immediately after oral challenge to measure the level of mMCP-1 and mMCP-7 (Figure 6 ). A sharp and significant decrease of both mMCP-1 and mMCP-7 was observed in EPIT mice compared to the sham group. Of note, the prolongation of the treatment period does not induce further decrease in the plasmatic concentration of these two proteases. Overall, these data indicate that EPIT to cashew can efficiently protect mice against IgE-mediated anaphylaxis induced by oral challenge.

\section{Discussion}

In this study, we aimed to evaluate the potential of EPIT to treat cashew allergy. To that end, we first developed a clinically relevant mouse model of anaphylaxis, for which $\operatorname{IgE}$ is the main mediator and in which challenge is performed by oral administration of allergens, similar to what occurs in humans. Using this model, we demonstrated that epicutaneous patches are able to deliver cashew allergens to skin DCs, especially Langerhans cells and $\mathrm{cDC} 2$ that have been demonstrated as the main promotors of tolerance to topical allergens. ${ }^{14,15}$ More importantly, EPIT with cashew patches affords a substantial level of protection against IgE-mediated anaphylaxis to cashew. In this study, we used a $50-\mu \mathrm{g}$ dose per patch. This dose was selected based upon preliminary experiments, showing that 100, 250 or $500 \mu \mathrm{g}$ doses did not afford additional benefits for protection (data not shown). This is, to our knowledge, the first study demonstrating the efficacy of a prototype immunotherapy against cashew allergy. Recently, Pereira et al. , evaluated the immunogenicity of orally-administered poly(anhydride) nanoparticles loaded with cashew allergens in BALB/c mice. ${ }^{16}$ These authors showed that nanoparticles were able to promote a strong specific Th1 response to cashew in association with an induction of FoxP3+ and LAP + T regulatory cells (T-Reg). Unfortunately, the clinical efficacy of this treatment has not been reported. In our preclinical model, we showed that EPIT induced a transient peak of cashew-specific IgE at the beginning of immunotherapy. Interestingly, the same phenomenon has been observed in patients during clinical trials investigating EPIT against peanut allergy. ${ }^{17}$ Moreover, and in a similar way to what has been observed in humans, this increase is followed 
by a gradual progressive decrease of IgE titers with continued treatment. Additionally, our results revealed that EPIT induces an increase of cashew-specific IgG (IgG1, mouse equivalent of human IgG4 and IgG2a, mouse equivalent of human IgG1). Again, these data should be viewed in the context of clinical data investigating EPIT for peanut allergy, in which a progressive increase of peanut specific IgG4 levels has been observed during the first 12 months of treatment. ${ }^{17}$ This increase in IgG4 response in humans is usually interpreted as a beneficial impact of the treatment since IgG4 antagonizes effector functions mediated by IgE through competition and neutralization of allergens. ${ }^{18}$ The permanent increase of IgG2a observed in our study should also be interpreted as a positive outcome since it is the main marker of Th1 immune orientation in mouse, which is known to be associated with non-allergic responses. ${ }^{19,20}$ Mast-cells express a high amount of high affinity IgE receptors (FcERI) and are unequivocally considered as key players in IgEdependent anaphylaxis. ${ }^{21}$ Additionally, it has been previously shown that IgE-dependent activation of mastcells plays an important role in disease induction in mouse models of allergy. ${ }^{22,23}$ Here, we demonstrated that EPIT strongly reduced mast-cell stimulation and degranulation following oral challenge. Interestingly, this inhibition occurs despite a relatively high level of $\operatorname{IgE}$, suggesting that EPIT modulates mast-cell reactivity to IgE signaling. Beyond the competition between $\operatorname{IgE}$ and $\operatorname{IgG}$ for allergen binding we described above, this modulation could be linked to an inhibition of the signal triggered by FceRI as described in previous studies. ${ }^{24,25}$ However, it is unlikely that this desensitization results from constant stimulation of gut mast-cell with small, sub-activating amounts of allergen, since EPIT does not lead to systemic diffusion of allergen. Therefore, we are currently performingin vivo experiments to elucidate the mechanisms underlying this modulation and the induction of tolerance to cashew, with a special focus on regulatory T-cells which have been demonstrated as key players in murine models of peanut allergy. ${ }^{26,27}$ Despite almost complete suppression of mMCP-1 and mMCP-7 levels in blood following 8 and 12 weeks of treatment, EPIT mice still presented substantial anaphylactic symptoms following oral challenge. Therefore, we cannot exclude a role for specific IgG in anaphylactic reactions, as suggested by Figure 2. Consequently, our mouse model may underestimate the potential efficacy of EPIT in humans, for which the role of IgG in anaphylaxis is still a matter of considerable debate.

EPIT may offer a safe approach to treat food allergy, ${ }^{28}$ primarily due to the absence of systemic dissemination of allergens, thus avoiding direct contact with anaphylaxis triggering effector cells, e.g. mast-cells. Therefore, we suggest that EPIT may be a relevant treatment strategy for cashew allergy. Indeed, the unique severity of reactions to cashew reported in allergic patients could be a source of concerns regarding other immunotherapeutic strategies that require subcutaneous injections or oral administration of allergens. A recent study demonstrated that many patients suffering from cashew allergy are IgE-sensitized to other nuts of the Anacardiaceae family. ${ }^{29}$ Thus, it would be interesting in future experiments to evaluate the capacity of cashew-specific EPIT to afford cross-protection against anaphylaxis to pistachio or mango, and therefore determine its spectrum of coverage among Anacardiaceae family members.

Overall, our data demonstrate that EPIT could be a relevant strategy to treat cashew allergy, bringing hope to patients suffering from this potentially life-threatening immune dysregulation.

\section{References}

1. Mendes C, Costa J, Vicente AA, Oliveira MBPP, Mafra I. Cashew Nut Allergy: Clinical Relevance and Allergen Characterisation. Clin Rev Allergy Immunol . 2019;57(1):1-22. doi:10.1007/s12016-016-8580-5

2. Johnson J, Malinovschi A, Alving K, Lidholm J, Borres MP, Nordvall L. Ten-year review reveals changing trends and severity of allergic reactions to nuts and other foods. Acta Paediatr Int J Paediatr . 2014;103(8). doi:10.1111/apa.12687

3. McWilliam V, Koplin J, Lodge C, Tang M, Dharmage S, Allen K. The Prevalence of Tree Nut Allergy: A Systematic Review. Curr Allergy Asthma Rep . 2015;15(54). doi:10.1007/s11882-015-0555-8

4. Crealey M, Alamin S, Tormey V, Moylett E. Clinical presentation of cashew nut allergy in a paediatric cohort attending an allergy clinic in the West of Ireland. Ir J Med Sci . 2019;188:219-222. doi:10.1007/s11845018-1801-6 
5. Clark AT, Anagnostou K, Ewan PW. Cashew nut causes more severe reactions than peanut: Case-matched comparison in 141 children.Allergy . 2007;62(8):913-916. doi:10.1111/j.1398-9995.2007.01447.x

6. Wang J, Sampson HA. Safety and efficacy of epicutaneous immunotherapy for food allergy. Pediatr Allergy Immunol . 2018;29(4):341-349. doi:10.1111/pai.12869

7. Mondoulet L, Dioszeghy V, Thébault C, Benhamou PH, Dupont C. Epicutaneous immunotherapy for food allergy as a novel pathway for oral tolerance induction. Immunotherapy . 2015;7(12). doi:10.2217/imt.15.86

8. Mondoulet L, Dioszeghy V, Puteaux E, et al. Intact skin and not stripped skin is crucial for the safety and efficacy of peanut epicutaneous immunotherapy (EPIT) in mice. Clin Transl Allergy . 2012;2(22). doi:10.1186/2045-7022-2-22

9. Hervé P-L, Dhelft V, Plaquet C, et al. Epidermal micro-perforation potentiates the efficacy of epicutaneous vaccination. J Control Release . 2019;298:12-26. doi:10.1016/j.jconrel.2019.02.004

10. Mattison CP, Malveira Cavalcante J, Izabel Gallão M, Sousa de Brito E. Effects of industrial cashew nut processing on anacardic acid content and allergen recognition by IgE. Food Chem . 2018;240:370-376. doi:10.1016/j.foodchem.2017.07.146

11. Mattison CP, Bren-Mattison Y, Vant-Hull B, Vargas AM, Wasserman RL, Grimm CC. Heatinduced alterations in cashew allergen solubility and IgE binding. Toxicol Reports . 2016;3:244-251. doi:10.1016/j.toxrep.2015.12.009

12. Brough HA, Liu AH, Sicherer S, et al. Atopic dermatitis increases the effect of exposure to peanut antigen in dust on peanut sensitization and likely peanut allergy. J Allergy Clin Immunol . 2015;135(1). doi:10.1016/j.jaci.2014.10.007

13. Finkelman FD. Anaphylaxis: Lessons from mouse models. J Allergy Clin Immunol . 2007;120(3):506-515. doi:10.1016/j.jaci.2007.07.033

14. Dioszeghy V, Mondoulet L, Laoubi L, et al. Antigen uptake by Langerhans cells is required for the induction of regulatory $\mathrm{T}$ cells and the acquisition of tolerance during epicutaneous immunotherapy in OVAsensitized mice. Front Immunol . 2018;9(1951). doi:10.3389/fimmu.2018.01951

15. Tordesillas L, Lozano-Ojalvo D, Dunkin D, et al. PDL2+ CD11b+ dermal dendritic cells capture topical antigen through hair follicles to prime LAP+ Tregs. Nat Commun . 2018;9(1). doi:10.1038/s41467-018-077167

16. Pereira MA, Rebouças J de S, Ferraz-Carvalho R de S, et al. Poly(anhydride) nanoparticles containing cashew nut proteins can induce a strong Th1 and Treg immune response after oral administration.Eur $J$ Pharm Biopharm . 2018;127:51-60. doi:10.1016/j.ejpb.2018.02.011

17. Sampson HA, Shreffler WG, Yang WH, et al. Effect of varying doses of epicutaneous immunotherapy vs placebo on reaction to peanut protein exposure among patients with peanut sensitivity: A randomized clinical trial. JAMA - J Am Med Assoc . 2017;318(18):1798-1809. doi:10.1001/jama.2017.16591

18. van de Veen W, Akdis M. Role of IgG4 in IgE-mediated allergic responses. J Allergy Clin Immunol . 2016;138(5):1434-1435. doi:10.1016/j.jaci.2016.07.022

19. Snapper CM, Paul WE. Interferon- $\gamma$ and B cell stimulatory factor-1 reciprocally regulate Ig isotype production. Science (80- ) . 1987;236(4804):944-947. doi:10.1126/science.3107127

20. Romagnani S. Immunologic influences on allergy and the TH1/TH2 balance. J Allergy Clin Immunol . 2004;113(3):395-400. doi:10.1016/j.jaci.2003.11.025

21. Galli SJ, Tsai M. IgE and mast cells in allergic disease. Nat Med . 2012;18(5):693-704. doi:10.1038/nm.2755 
22. Mayr SI, Zuberi RI, Liu FT. Role of immunoglobulin E and mast cells in murine models of asthma. Brazilian J Med Biol Res . 2003;36(7):821-827. doi:10.1590/S0100-879X2003000700001

23. van Halteren AGS, van der Cammen MJF, Biewenga J, Savelkoul HFJ, Kraal G. IgE and mast cell responses on intestinal allergen exposure: A murine model to study the onset of flood allergy. J Allergy Clin Immunol . 1997;99(1):94-99. doi:10.1016/S0091-6749(97)70305-1

24. Gladys Ang WX, Church AM, Kulis M, Choi HW, Wesley Burks A, Abraham SN. Mast cell desensitization inhibits calcium flux and aberrantly remodels actin. J Clin Invest . 2016;126(11):4103-4118. doi:10.1172/JCI87492

25. Oka T, Rios EJ, Tsai M, Kalesnikoff J, Galli SJ. Rapid desensitization induces internalization of antigen-specific IgE on mouse mast cells. J Allergy Clin Immunol . 2013;132(4):922-932. doi:10.1016/j.jaci.2013.05.004

26. Dioszeghy V, Mondoulet L, Puteaux E, et al. Differences in phenotype, homing properties and suppressive activities of regulatory $\mathrm{T}$ cells induced by epicutaneous, oral or sublingual immunotherapy in mice sensitized to peanut. Cell Mol Immunol . 2017;14(9):770-782. doi:10.1038/cmi.2016.14

27. Tordesillas L, Mondoulet L, Blazquez AB, Benhamou PH, Sampson HA, Berin MC. Epicutaneous immunotherapy induces gastrointestinal LAP + regulatory T cells and prevents food-induced anaphylaxis. $J$ Allergy Clin Immunol . 2017;139(1):189-201. doi:10.1016/j.jaci.2016.03.057

28. Kim EH, Burks AW. Food allergy immunotherapy: OIT and EPIT.Allergy . Published online 2020. doi:https://doi.org/10.1111/all.14220

29. Bastiaan-Net S, Reitsma M, Cordewener JHG, et al. IgE Cross-Reactivity of Cashew Nut Allergens. Int Arch Allergy Immunol . 2019;178(1):19-32. doi:10.1159/000493100

\section{Legend to the figures}

Figure 1: Development and characterization of mouse model of cashew sensitization and anaphylaxis. Mice were sensitized to cashew through skin (in red) or oral (in green) routes. As a negative control, a group of naïve mice (in white) was included. (A ) Cashew-specific IgE, IgG1 and IgG2a antibody responses were evaluated by ELISA, from blood samples collected one week after the end of the sensitization. Mice were orally challenged to cashew one week after the end of the sensitization. (B ) Body temperature was measured every 5 minutes following challenge for 60 minutes. (C) Area under the curve was calculated for each individual percentage of temperature variation curve using $100 \%$ as a baseline. mMCP-1 (D) and mMCP-7 (E ) concentrations were measured by ELISA from plasma collected immediately after the challenge, $(\mathrm{n}=8$ per experimental group). Data are median with interquartile range of individual values. $\mathrm{P}$ values were determined using the Mann-Whitney unpaired t-test (**, $\mathrm{p}<0,01 ; * * *, \mathrm{p}<0,001 ;$ n.s., non-significant). For $\mathbf{B}, \mathbf{C}, \mathbf{D}$ and $\mathbf{E}$ panels, the level of significant measured between each sensitized group and the negative control group is indicated above each graph.

Figure 2: Validation of the oral route of sensitization as a trigger of IgE-mediated anaphylaxis in mice. Mice were orally sensitized to cashew. One week after the end of the sensitization, mice received IgE blocking antibody (clone EM-95, in green), IgG blocking antibody (anti-FcrRII/RIII clone 2.4G2, in blue) or relevant isotype controls (in red). The day after, mice were challenged orally to cashew. (A ) Body temperature was measured every 5 minutes following challenge for 60 minutes and (B) diarrhea occurrence and severity was scored. mMCP-1 (C) and mMCP-7 (D ) concentrations were measured by ELISA from plasma collected immediately after the challenge, $(\mathrm{n}=8$ per experimental group). Data are median with interquartile range of individual values. $\mathrm{P}$ values were determined using the Mann-Whitney unpaired $\mathrm{t}$ test $\left(^{*}, \mathrm{p}<0,05 ;{ }^{* * *}, \mathrm{p}<0,001\right.$; n.s., non-significant). For $\mathrm{C}$ and $\mathrm{D}$ panels, the level of significant measured between each sensitized group and the negative control group is indicated above each dot plot.

Figure 3: Cashew allergens are efficaciously delivered by epicutaneous patches, leading to allergen capture by skin dendritic cells. Mice were orally sensitized to cashew. One week after the end 
of the sensitization, mice received a patch loaded with cashew protein extract conjugated to Fluoroprobe-647 (F-647) for 48 hours. As negative controls, mice received a patch containing excipient. Brachial draining lymph nodes were collected, and cells were isolated and labelled for FACS analysis. The absolute number of cashew-positive (F-647-positive) cells was measured among each DC subsets, $(\mathrm{n}=7-8$ per experimental group). Data are Median and interquartile ranges of individual values. $\mathrm{P}$ values were determined according to the Mann-Whitney test $\left({ }^{* *}, \mathrm{p}<0.01 ;{ }^{* * *}, \mathrm{p}<0.001\right)$.

Figure 4: Kinetic modulation of cashew-specific antibody response following EPIT to cashew nut in mice. Mice were orally sensitized to cashew. One week after the end of the sensitization, mice were submitted to EPIT. To that end, mice received cashew patches containing $50 \mu \mathrm{g}$ of cashew protein extract, once a week for up to 16 weeks (in blue). Patches were applied for 48 hours. As negative controls, mice received patches containing excipient (sham, in black) or were kept untreated (naïves, in white). Blood samples were collected before EPIT (B) or every two weeks during treatment to isolate plasma (weeks 2, 4, $6,8,10,12,14,16)$, as indicated on $\mathrm{C}$ panel. Cashew-specific antibody titers were measured from plasma by indirect ELISA (IgE, panel A ) or direct ELISA (IgG1 and IgG2a, panelB and panel C , respectively), $(\mathrm{n}=8$-24 per experimental group -8 mice of each group were challenged at weeks 8 and 12 and sacrificed). Data are Median and interquartile ranges of individual values.

Figure 5: Evaluation of the level of protection afforded by EPIT against anaphylaxis following 8,12 or 16 weeks of treatment . Mice were orally sensitized to cashew and treated as described in Figure 4. Following 8 (A, B ), $12(\mathbf{C}, \mathbf{D})$ or $16(\mathbf{E}, \mathbf{F})$ weeks of EPIT, 8 mice of each group were challenged orally to cashew. (A, C, E ) Body temperature was measured every 5 minutes following challenge for 60 minutes. Data are Median and interquartile ranges of individual values. (B, D, F ) Clinical symptoms were monitored every 5 minutes following challenge for 60 minutes. ( $\mathrm{n}=8$ per experimental group). Data are Mean with SEM of individual values. $\mathrm{P}$ values were determined according to the Mann-Whitney test.

Figure 6: Evaluation of mast-cell activation induced by oral challenge following 8,12 or 16 weeks of EPIT . Mice were orally sensitized to cashew, treated as described in Figure 4 and finally challenged as described in Figure 5. Blood samples were collected 60 minutes after the challenge to isolate plasma. mMCP-1 (A, C, E ) and mMCP-7 (B, D, F ) concentrations were measured from plasma by ELISA, ( $\mathrm{n}=8$ per experimental group). Data are median with interquartile range of individual values. $\mathrm{P}$ values were determined using the Mann-Whitney unpaired t-test $(* *, \mathrm{p}<0,01 ; * * *, \mathrm{p}<0,001)$.

\section{Hosted file}

Figures.pdf available at https://authorea.com/users/341513/articles/468402-epicutaneousimmunotherapy-protects-cashew-sensitized-mice-from-anaphylaxis

\section{SUPPLEMENTARY}

\section{Supplementary legends to the figures}

Figure S1: Analysis of on-site prepared cashew protein extracts by SDS PAGE. Protein fraction was extracted from defatted cashew flour as indicated in material and methods. This extract was denatured and analyzed by SDS PAGE (lane 2) in comparison to initial cashew flour (lane 1) and commercial cashew protein extract (Stallergenes Greer, lane 3). The relative molecular mass (kDa) is indicated on the left. The position of major allergens are indicated on the right, based on previous published data. Note that Ana o 2 separates as two distinct bands following denaturation since it is constituted by two subunits (acidic and basic) linked by disulfide bonds.

Figure S2: Analysis of cashew protein extracts recovered from patches. Epicutaneous patches were loaded with commercial cashew protein extracts (Stallergenes Greer) in liquid form, by dissolving protein lyophilizate in PBS 1X, NaCl $0.45 \%$ or phosphate buffer $0.1 \mathrm{M}$. Patches were dried for one hour at $30^{\circ} \mathrm{C}$. Then, proteins were redissolved from patch backing using distilled water. (A) Recovered proteins were used 
as a coating in ELISA to analyze their capacity to bind to cashew-specific antibodies induced in mice by oral sensitization. HRP-conjugated anti-mouse IgG1 (left panel) or anti-mouse IgE (right panel) were used as secondary antibodies. Optical densities (O.D.) at $450 \mathrm{~nm}$ were plotted against plasma dilution, $(\mathrm{n}=7$ per group). Data are median and range of individual values and non-linear regression curve was obtained using sigmoidal 4PL equation. (B ) Recovered protein extracts were centrifuged and total extract (before centrifugation, $\mathrm{T})$, supernatant $(\mathrm{S})$ and pellet $(\mathrm{P})$ were denatured and analyzed by SDS PAGE. The relative molecular mass $(\mathrm{kDa})$ is indicated on the left.

\section{Supplementary methods}

\section{Analysis of cashew protein extract by SDS PAGE.}

Samples were prepared in Laemmli buffer and denatured 10 minutes at $95{ }^{\circ} \mathrm{C}$. Denatured samples were separated on $12 \%$ polyacrylamide gel (Criterion TGX, Bio-Rad) using Tris-Glycine running buffer. Proteins were detected by Coomassie blue (SimplyBlue ${ }^{\mathrm{TM}}$ SafeStain, Invitrogen). Novex Sharp Prestained Protein Strandard (Invitrogen) was used as a ladder.

\section{Analysis of patch content by SDS PAGE and ELISA.}

Epicutaneous patches were loaded with commercial cashew protein extract (Stallergenes Greer, Lenoir, NC) prepared in PBS 1X, NaCl $0.45 \%$ or phosphate buffer $0.1 \mathrm{M}$ and dried as described above. Dry deposition was solubilized in distilled water and centrifugated 5 minutes at $2000 \mathrm{x}$ g. The solutions obtained before centrifugation, as well as supernatants and pellets were analyzed by SDS-PAGE as described above or by ELISA. For ELISA, 96-well plates were coated with proteins recovered from patches $(5 \mu \mathrm{g} / \mathrm{mL}$, protein equivalent) and incubated overnight at $4{ }^{\circ} \mathrm{C}$. Plates were saturated with PBS 1X; Tween ${ }^{\circledR} 0.05 \%$; BSA $1 \%$ for 1 hour at room temperature (RT). Plates were incubated with serially diluted plasma collected from orally sensitized mice for 2 hours at RT, then with HRP-conjugated goat anti-mouse IgG1 or IgE (Invitrogen) diluted 1:10000 or 1:1000, respectively, for 1 hour at RT. HRP was revealed using TMB substrate (eBioscience), for 15 minutes at RT. The reaction was stopped with $1 \mathrm{~N}$ chloride acid and the optical density (O.D.) at $450 \mathrm{~nm}$ was recorded. 
Figure 1

A

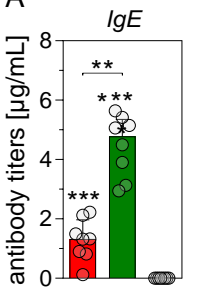

$\mathrm{C}$

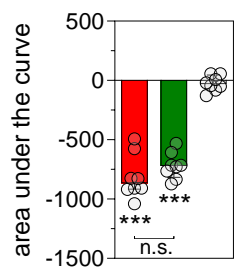

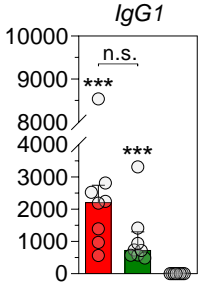

D

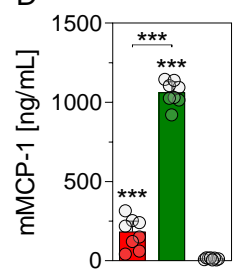

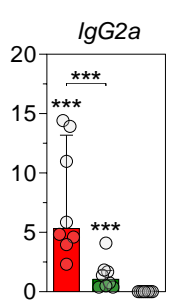

$\mathrm{E}$
B

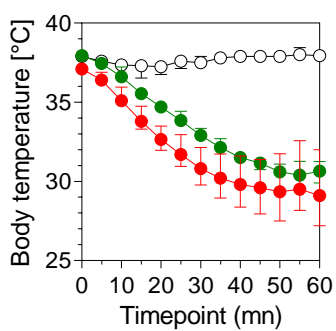

Timepoint (mn)

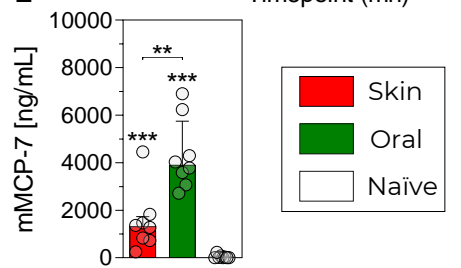

Figure 1: Development and characterization of mouse model of cashew sensitization and anaphylaxis. Mice were sensitized to cashew through skin (in red) or oral (in green) routes. As a negative control, a group of naïve mice (in white) was included. (A) Cashew-specific IgE, IgG1 and IgG2a antibody responses were evaluated by ELISA, from blood samples collected one week after the end of the sensitization. Mice were orally challenged to cashew one week after the end of the sensitization. (B) Body temperature was measured every 5 minutes following challenge for 60 minutes. (C) Area under the curve was calculated for each individual percentage of temperature variation curve using $100 \%$ as a baseline. mMCP-1 (D) and mMCP-7 (E) concentrations were measured by ELISA from plasma collected immediately after the challenge, $(n=8$ per experimental group). Data are median with interquartile range of individual values. $P$ values were determined using the Mann-Whitney unpaired t-test $\left(^{\star \star}, p<0,01 ;{ }^{* \star}, p<0,001\right.$; n.s., non-significant). For $B$, $\mathrm{C}, \mathrm{D}$ and $\mathrm{E}$ panels, the level of significant measured between each sensitized group and the negative control group is indicated above each graph. 